\title{
Monitoring disease progression with plasma creatinine in amyotrophic lateral sclerosis clinical trials
}

\author{
Ruben P A van Eijk, ${ }^{1}$ Marinus J C Eijkemans, ${ }^{2}$ Toby A Ferguson, ${ }^{3}$ \\ Stavros Nikolakopoulos, ${ }^{2}$ Jan $\mathrm{H}$ Veldink, ${ }^{1}$ Leonard $\mathrm{H}$ van den Berg ${ }^{1}$
}

\begin{abstract}
- Additional material is published online only. To view please visit the journal online (http://dx.doi.org/10.1136/ jnnp-2017-317077)
\end{abstract}

${ }^{1}$ Department of Neurology, University Medical Center Utrecht, Utrecht, The Netherlands

${ }^{2}$ Department of Biostatistics and Research Support, University Medical Center Utrecht, Utrecht, The Netherlands

${ }^{3}$ Biogen Idec, Boston,

Massachusetts, USA

Correspondence to Professor Leonard $\mathrm{H}$ van den Berg, Department of Neurology, University Medical Center Utrecht, Heidelberglaan 100, 3584 CX, The Netherlands; I.h. vandenBerg@umcutrecht.nl

Received 14 August 2017 Revised 25 September 2017 Accepted 4 October 2017 Published Online First 30 October 2017
ABSTRACT

Objectives Plasma creatinine is a predictor of survival in amyotrophic lateral sclerosis (ALS). It remains, however, to be established whether it can monitor disease progression and serve as surrogate endpoint in clinical trials.

Methods We used clinical trial data from three cohorts of clinical trial participants in the LITRA, EMPOWER and PROACT studies. Longitudinal associations between functional decline, muscle strength and survival with plasma creatinine were assessed. Results were translated to trial design in terms of sample size and power.

Results A total of 13564 measurements were obtained for 1241 patients. The variability between patients in rate of decline was lower in plasma creatinine than in ALS functional rating scale-Revised (ALSFRS-R; $p<0.001$ ). The average rate of decline was faster in the ALSFRS-R, with less between-patient variability at baseline $(p<0.001)$. Plasma creatinine had strong longitudinal correlations with the ALSFRS-R $(0.43(0.39-0.46)$, $p<0.001)$, muscle strength $(0.55(0.51-0.58), p<0.001)$ and overall mortality (HR 0.88 (0.86-0.91, p<0.001)). Using plasma creatinine as outcome could reduce the sample size in trials by $21.5 \%$ at 18 months. For trials up to 10 months, the ALSFRS-R required a lower sample size.

Conclusions Plasma creatinine is an inexpensive and easily accessible biomarker that exhibits less variability between patients with ALS over time and is predictive for the patient's functional status, muscle strength and mortality risk. Plasma creatinine may, therefore, increase the power to detect treatment effects and could be incorporated in future ALS clinical trials as potential surrogate outcome.

\section{INTRODUCTION}

\section{SLinked}

- http://dx.doi.org/10.1136/ jnnp-2017-317413

\section{CrossMark}

\section{To cite: van Eijk RPA,}

Eijkemans MJC, Ferguson TA, et al. J Neurol Neurosurg

Psychiatry 2018;89:156-161.
One of the challenging issues in the search for drug therapy in amyotrophic lateral sclerosis (ALS) is disease heterogeneity. The ALS functional rating scale-revised (ALSFRS-R) has become the standard to evaluate ALS disease progression. The ALSFRS-R is, however, a subjective instrument and insensitive to small alterations in disease progression. Moreover, patients with the same score may not represent the same severity of ALS, which may further inflate between-patient differences. ${ }^{1-3}$ Biological markers, or biomarkers, are, therefore, of interest for ALS research and could potentially improve clinical trial design. Surrogate biomarkers for disease progression could detect treatment effects more objectively, potentially reducing follow-up durations and sample size. ${ }^{4}$ Furthermore, not awaiting fatal endpoints such as death or tracheostomy-free survival would make trials more efficient. $^{5}$

Plasma creatinine is a breakdown product of creatine phosphate in muscles and is related to muscle mass. ${ }^{6-9}$ Although several studies have demonstrated reduced plasma creatinine levels in patients with $\mathrm{ALS}^{10-14}$ and the prognostic value for survival ${ }^{11}{ }^{15-17}$ and functional decline at baseline, ${ }^{91819}$ a strong longitudinal evidence for a direct relationship with mortality, functional decline and muscle strength is still lacking. Moreover, it remains to be established whether plasma creatinine might be a good candidate to serve as surrogate endpoint in ALS clinical trials.

The aim of this study is, therefore, to determine the value of plasma creatinine, compared with other classical endpoints, in monitoring disease progression during clinical trials, in a large, well-defined cohort of trial participants.

\section{METHODS}

\section{Study population}

Data for this study originated from three independent cohorts: the LITRA study, EMPOWER study and the PROACT database. The LITRA study was a randomised, placebo-controlled trial to study the efficacy of lithium carbonate on survival in ALS. Details regarding the study design, intervention and results have been reported elsewhere. ${ }^{20}$ Neither survival nor ALSFRS-R showed a beneficial effect of lithium carbonate in patients with ALS. To validate our results, we obtained data from a second trial cohort who participated in the EMPOWER study. This was a large $(n=942)$ international multicentre study to determine the efficacy of dexpramipexole. ${ }^{21}$ Unfortunately, neither survival nor ALSFRS-R showed a beneficial effect of dexpramipexole in patients with ALS. A second validation cohort was obtained from the PRO-ACT database (version December 2015), which consists of 23 trials executed over the past 20 years. ${ }^{15}$ The PRO-ACT database is Institutional Review Board approved, using solely anonymised data. Subjects within the database are not traceable; they consented to participate during the individual trials. The process of converting PRO-ACT data, so that it 
is suitable for analysis, is described in the online supplementary eMethods.

Clinical follow-up data were matched with laboratory and survival data. Plasma creatinine levels were time matched with an ALSFRS-R score. Ideally, the ALSFRS-R score was acquired on the same day. If no ALSFRS-R score was available on that day, the closest score within 15 days was taken; alternatively, the ALSFRS-R was recorded as missing. Only patients with at least two matched clinical and laboratory follow-up moments were included to accurately determine their mutual and individual relationship with disease progression. Missing data in baseline demographics, clinical characteristics and survival (defined as time to death from any cause) were manually extracted from the electronic hospital information system for the LITRA participants and were fully imputed. One patient with highly elevated plasma creatinine values due to renal disease was excluded from the analysis.

\section{Statistical analysis}

The longitudinal relationships over time and between the ALSFRS-R total score and plasma creatinine level were analysed using linear mixed effect models (LMEs). Both the ALSFRS-R and plasma creatinine were standardised to make direct comparisons possible. A base model, containing a fixed effect of time and a random intercept per individual, was supplemented with a random slope for time to assess whether there are differences in the rate of decline between patients (as measure of disease heterogeneity or between-patient slope variability). To test whether the amount of slope variability was different for the ALSFRS-R and plasma creatinine, all outcome data were merged and an indicator variable (ALSFRS-R or creatinine) was created. An LME was fitted using the merged outcomes with a random slope for the indicator variable, random slope for time and random slope for the interaction between the indicator variable and time. The random interaction term in this merged model indicates whether the differences between patients in rate of decline are different for plasma creatinine and the ALSFRS-R. From the separate mixed models, we extracted the best-unbiased linear predictions (BLUP) of the rate of decline for the ALSFRS-R and plasma creatinine per individual. The relationships between the ALSFRS-R, plasma creatinine and survival were assessed within a joint modelling framework. ${ }^{22}$ The joint models were fitted with a piecewise-constant baseline risk function for survival time. Independent predictors of survival were selected based on a stepwise backward approach using the likelihood ratio test (LR test). LMEs were fitted with maximum likelihood when comparisons were made between models. If variances, coefficients or BLUPs were determined, a restricted likelihood estimation was applied. Results were considered significant when the $\mathrm{p}$ value was lower than 0.05. Linear mixed models were fitted using the lmer function in the $\mathrm{R}$ package lme4 (version 1.1-12); CIs for the variances were estimated using the profile function. ${ }^{23}$ Longitudinal sample size calculations were performed according to Edland (2009) with the Immpower function. ${ }^{24}$ Joint models were fitted using the R package JM (version 1.4-5). ${ }^{22}$

\section{RESULTS}

\section{Study population}

A total of 13564 ALSFRS-R and plasma creatinine measurements were obtained for 1241 patients; the cumulative follow-up time was 1292 years (table 1). Patients participating in the EMPOWER study were subdivided into two cohorts according to the original scale of plasma creatinine $(\mu \mathrm{mol} / \mathrm{L}$ vs $\mathrm{mg} / \mathrm{dL}$, supplementary eFigure 1). In our sample, $44.7 \%(n=555)$ of the patients had a low plasma creatinine level at baseline (males below $74 \mu \mathrm{mol} / \mathrm{L}$, and females below $56 \mu \mathrm{mol} / \mathrm{L}$ ).

\section{Rate of decline and heterogeneity in disease progression between subjects}

The ALSFRS-R total score and the plasma creatinine levels declined significantly over time in all cohorts (all $\mathrm{p}<0.001$; LR test, figure 1). The SD of the rate of decline between patients was lower for plasma creatinine (0.04 (CI: 0.04 to 0.05$)$ ) than for the ALSFRS-R (0.10 (CI: 0.09 to 0.10$))(p<0.001)$, indicating more between-patient variability in rates of decline in ALSFRS-R scores. The average rate of decline was, however, faster in the ALSFRS-R, with less between-patient variability at baseline $(\mathrm{p}<0.001)$. These findings were identical among cohorts (see online supplementary eTable 1). Patients with a high rate of decline on ALSFRS-R had a high rate of decline in plasma creatinine (Spearman $r=0.47$ (CI: 0.42 to 0.53 ), $\mathrm{p}<0.001$ ).

Table 1 Baseline demographics and clinical characteristics of study participants in LITRA, EMPOWER and PRO-ACT

\begin{tabular}{|c|c|c|c|c|}
\hline \multirow[b]{2}{*}{ Demographic } & \multirow[b]{2}{*}{ LITRA $(n=50)$} & \multicolumn{2}{|l|}{ EMPOWER } & \multirow[b]{2}{*}{ PRO ACT $(n=255)$} \\
\hline & & $\mu \mathrm{mol} / \mathrm{L}(\mathrm{n}=449)$ & $\mathrm{mg} / \mathrm{d} L(\mathrm{n}=487)$ & \\
\hline Age, years & $56(11)$ & $57(12)$ & $57(11)$ & $57(11)$ \\
\hline Site of symptom onset (bulbar) & $13(26 \%)$ & $103(23 \%)$ & $116(24 \%)$ & $58(23 \%)$ \\
\hline Disease duration from symptom onset, months & $15(8)$ & $15(5)$ & $15(5)$ & $15(6)$ \\
\hline FVC (\% predicted) & $99(17)$ & $90(17)$ & $88(18)$ & $92(17)$ \\
\hline$\triangle \mathrm{FRS}$, months* & $-0.57(1.6)$ & $-0.56(1.6)$ & $-0.68(1.6)$ & $-0.60(1.6)$ \\
\hline Plasma creatinine level $(\mu \mathrm{mol} / \mathrm{L})$ & $74(14)$ & $69(15)$ & $72(17)$ & $68(15)$ \\
\hline Male & $79(13)$ & $74(15)$ & $77(16)$ & $73(15)$ \\
\hline Female & $64(11)$ & $60(11)$ & $61(13)$ & $61(13)$ \\
\hline Bulbar onset & $74(9)$ & $71(14)$ & $74(16)$ & $69(13)$ \\
\hline Spinal onset & $74(15)$ & $69(16)$ & $71(17)$ & $68(16)$ \\
\hline
\end{tabular}

Data are mean (SD) or $\mathrm{n}(\%)$.

${ }^{*} \triangle \mathrm{FRS}=($ ALSFRS-R score at inclusion -48$)$ /disease duration from symptom onset.

ALSFRS-R, amyotrophic lateral sclerosis functional rating scale-revised; FVC\%, forced vital capacity. 
LITRA - ALSFRS-R
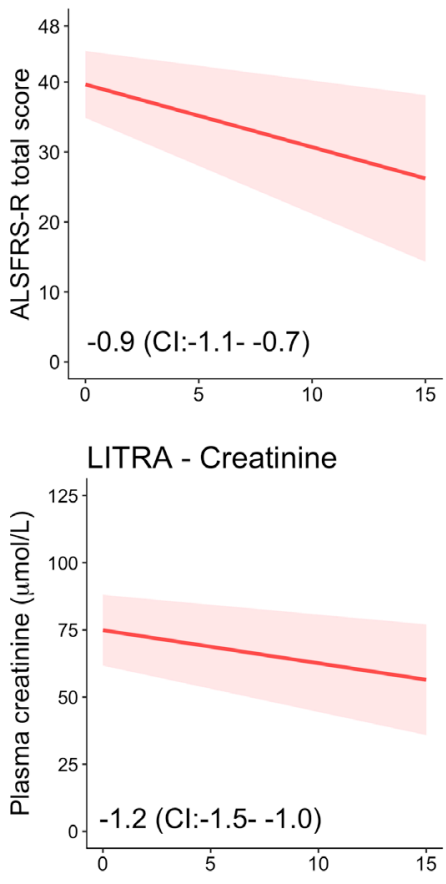

EMPOWER - ALSFRS-R

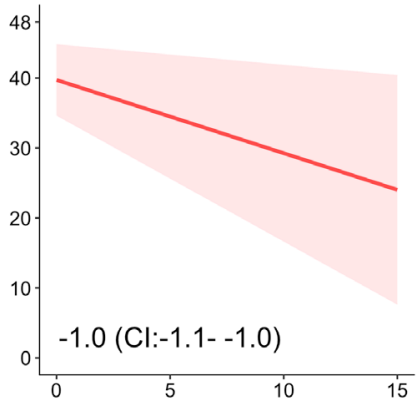

EMPOWER - Creatinine

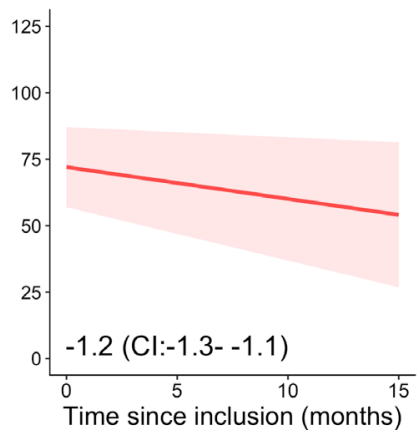

PROACT - ALSFRS-R

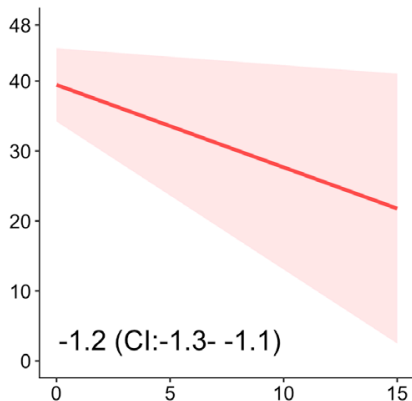

PROACT - Creatinine

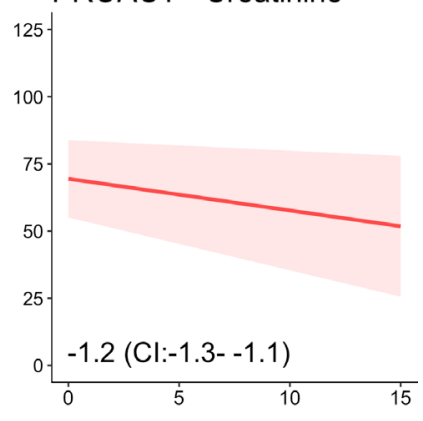

Figure 1 Longitudinal decline in ALSFRS-R total score and plasma creatinine level. Matched longitudinal ALSFRS-R and laboratory data from 50 patients in the LITRA study, 936 patients in the EMPOWER study and 255 patients in the PROACT database. The red solid line is the mean rate of decline over time. Both the ALSFRS-R and plasma creatinine declined significantly over time (all $p<0.001$; LR test). The red shaded area is 1 SD around the mean rate of decline and represents the variability between patients over time. Note the severe funnelling out in ALSFRS-R total scores and the more homogenous pattern of decline in plasma creatinine, best seen in the EMPOWER and PROACT cohorts. ALSFRS-R, amyotrophic lateral sclerosis functional rating scale-revised; LR test, likelihood ratio test.

This relationship was more prominent in the motor items of the ALSFRS-R (Spearman $r=0.49$ (CI: 0.43 to 0.54 ), $\mathrm{p}<0.001$ ) than in the bulbar items (Spearman $r=0.30$ (CI: 0.24 to 0.37 ), $\mathrm{p}<0.001$ ). The respiratory items had a Spearman $r$ of 0.31 (CI: 0.24 to 0.37$), p<0.001$. In contrast, the between-patient variability in rates of decline in ALSFRS-R scores was reduced within the separate bulbar and motor subdomains (0.08 (CI: 0.07 to 0.08 ) and 0.07 (CI: 0.07 to 0.08$)$, both $\mathrm{p}<0.001)$, but increased in the respiratory subdomain $(0.12(0.12-0.13), \mathrm{p}<0.001)$, as compared with the total score.

Plasma creatinine, muscle strength and slow vital capacity in EMPOWER

During a 12-month period, there was an approximate linear relationship between the mean level of plasma creatinine and the mean muscle strength, with an overall longitudinal correlation of 0.55 (CI: 0.51 to $0.58, \mathrm{p}<0.001$; LR test, figure 2 ). Interestingly, for the first 2 months in the $\mu \mathrm{mol} / \mathrm{L}$ group, there is a relatively stable mean level of plasma creatinine, while muscle strength is deteriorating. This effect is, however, not seen in the $\mathrm{mg} / \mathrm{dL}$ group. Neither in the PRO-ACT data nor in the LITRA study is a plateau phase identified, indicating that the $\mu \mathrm{mol} / \mathrm{L}$ group may show a possible measurement error (ie, difference between fasted or non-fasted blood draw during enrolment). The longitudinal correlation between slow vital capacity and plasma creatinine was considerably lower $(0.32$ (CI: 0.28 to 0.36), $\mathrm{p}<0.001$; LR test).

Longitudinal relationship mortality, plasma creatinine level and ALSFRS-R total score

Overall, mortality at 18 months after inclusion was $26.8 \%$ (CI: $21.4 \%$ to $31.9 \%$ ). Baseline $\Delta \mathrm{FRS},{ }^{25}$ plasma creatinine, vital capacity, age, gender and site of onset were independent predictors of survival and were adjusted for in subsequent models (see online supplementary eTable 2). The adjusted HR

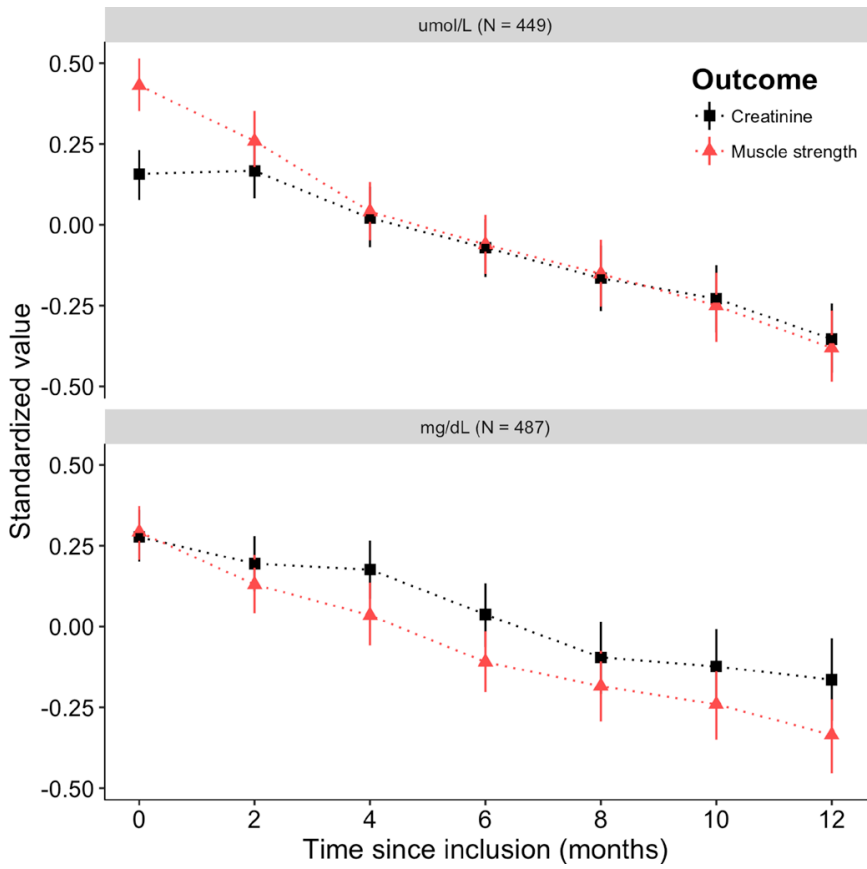

Figure 2 Longitudinal relationship between muscle strength and plasma creatinine during a 12-month follow-up period. Mean values with bootstrapped $95 \% \mathrm{Cls}$ are given for plasma creatinine and muscle strength at bimonthly follow-up moments for the first 12 months in the EMPOWER study. 
Table 2 Longitudinal associations of ALSFRS-R total score and plasma creatinine levels with mortality during follow-up

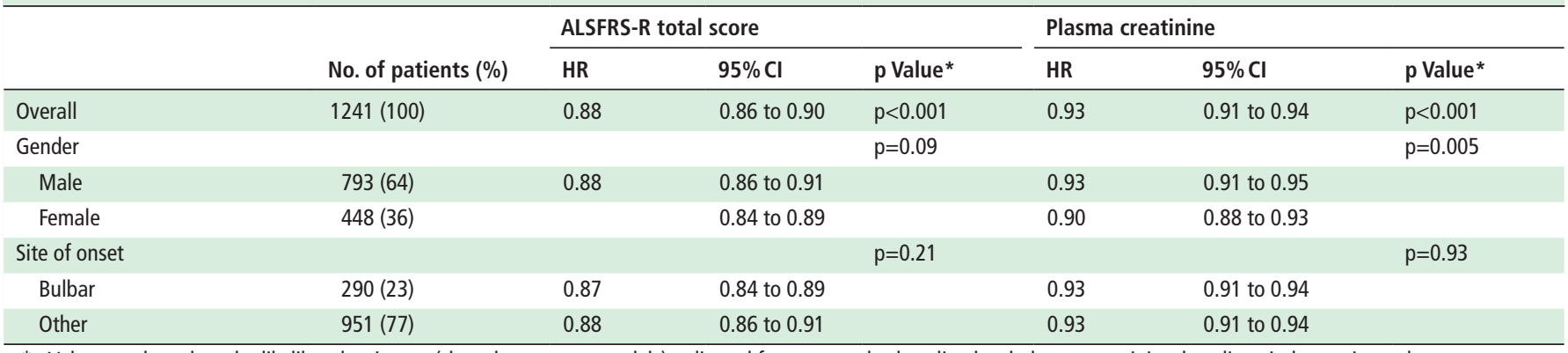

* $p$ Values are based on the likelihood ratio test (shared parameter models), adjusted for age, gender, baseline level plasma creatinine, baseline vital capacity and $\triangle$ FRS. Participants were followed for a maximum of 31.9 months (median 14.0 months).

ALSFRS-R, amyotrophic lateral sclerosis functional rating scale-revised.

of having a low plasma creatinine at baseline was 1.56 (CI: 1.20 to 2.03). Both the longitudinal ALSFRS-R total score and plasma creatinine level strongly affected the risk of mortality (table 2). The site of symptom onset did not affect the risk of mortality for either the ALSFRS-R total score or for the plasma creatinine level $(p=0.21$ and $p=0.92$, respectively; LR test). On the other hand, a rise in plasma creatinine levels affected the risk of mortality during follow-up more strongly in females than in males $(p=0.005$; LR test $)$.

\section{Trial design using plasma creatinine}

Figure 3 shows how sample size is affected if either the ALSFRS-R or plasma creatinine is taken as primary outcome. For short trials (up to 10 months), the smaller variability between patients at baseline and relatively faster average rate of decline of the ALSFRS-R (figure 1) resulted in a smaller sample size. For trials longer than 11 months, the lower variability between patients in rate of decline in plasma creatinine positively affected the sample size. At 18 months, the sample size is $21.5 \%$ smaller when using plasma creatinine as outcome rather than the ALSFRS-R ( $n=153$ vs $n=195$, figure $3 A$ ). Changing the study design to bimonthly visits resulted in small increases in sample size for the ALSFRS-R, while for plasma creatinine, this change considerably affected the sample size due to the higher intrapatient variability (see online supplementary eTable 1 ). Figure 3B shows how sample size is inflated when using $\mathrm{mg} / \mathrm{dL}$ due to reduced precision (see online supplementary eFigure 1). Although plasma creatinine is capable of measuring disease progression in both spinal and bulbar-onset patients, the utility of plasma creatinine seems higher for spinal-onset patients (figure 3C and D).

\section{DISCUSSION}

Our results fill an important gap in the literature regarding the value of plasma creatinine for monitoring ALS disease progression during clinical trials. Heterogeneity in the rate of decline between patients negatively influences the power to detect treatment effects. We show that there is considerably less between-patient variability in rates of decline of plasma creatinine than of the ALSFRS-R. This is a significant result because it directly affects the power to detect treatment effects during clinical trials. As shown in figure $3 \mathrm{~A}$, even though the ALSFRS-R has, on average, a faster rate of decline, with less variability at baseline, when the follow-up duration exceeds 10 months, plasma creatinine requires fewer patients, or is able to detect a smaller treatment effect, with an identical sample size compared with the ALSFRS-R.

Our results show that the patients' current level of plasma creatinine correlates with the risk of death and indicate a strong longitudinal relationship between mortality and plasma creatinine loss. Previous studies have shown that the baseline measurement of plasma creatinine is a strong predictor of survival. ${ }^{11} 15-17$ The reported HRs vary between studies (ranging from 1.2 to 1.5), but consistently show an increased risk of death if levels of plasma creatinine are low; this is in line with our results (low baseline plasma creatinine HR 1.56 (CI: 1.20 to 2.03)). However, as plasma creatinine levels change over time and a fixed level from baseline cannot be assumed, our longitudinal assessment appears to be more relevant for determining the direct relationship between the risk of dying and creatinine loss.

Heterogeneity in rates of decline is inevitable in ALS due to its divergent prognosis. However, the increased between-patient variability in rate of decline of the ALSFRS-R could be due to the internal construction of the ALSFRS-R score: as patients with the same score may not represent the same severity of ALS, this may influence the rate of decline. ${ }^{13}$ Our analysis of the subgroups supports this hypothesis, revealing that separating the motor and bulbar subdomains from the total score, between-patient variability in rates of decline can be reduced, although between-patient variability remains higher than in plasma creatinine. Plasma creatinine level is most likely related to muscle mass; it could, therefore, be seen as marker of affected muscle mass and may thus better approximate ALS disease severity than the ALSFRS-R, potentially reducing the heterogeneity in rates of decline. ${ }^{6-9}$ Our results show that muscle strength and plasma creatinine levels are strongly correlated, and underscore the potential relationships between muscle mass, muscle strength and plasma creatinine levels. Because the patients' overall muscle strength is well correlated with disease progression, ${ }^{26}$ plasma creatinine may provide additional information or reduce the number of tested muscle groups and potentially reduce the patients' burden when participating in ALS clinical trials.

A major benefit of plasma creatinine is that it is easy and inexpensive to determine, improving its applicability for international multicentre trials without the need for specific laboratory equipment or invasive cerebrospinal fluid access. It is unlikely that plasma creatinine reflects a pathological process or that it can be used as a pharmacodynamic biomarker, like, for example, SOD1 protein in CSF in a recent clinical trial. ${ }^{27}$ It is, therefore, unlikely that plasma creatinine can detect disease activity in very early stages of ALS (ie, re-innervation with preserved muscle mass). Our results reveal that plasma creatinine may have less power to detect a treatment effect than the ALSFRS-R for early phase I/II exploratory trials with follow-up less than 10 months and, due to its biological variability, a higher sampling rate (preferably monthly) would be required (figure $3 \mathrm{~A}$ ). Nevertheless, as plasma creatinine is a 

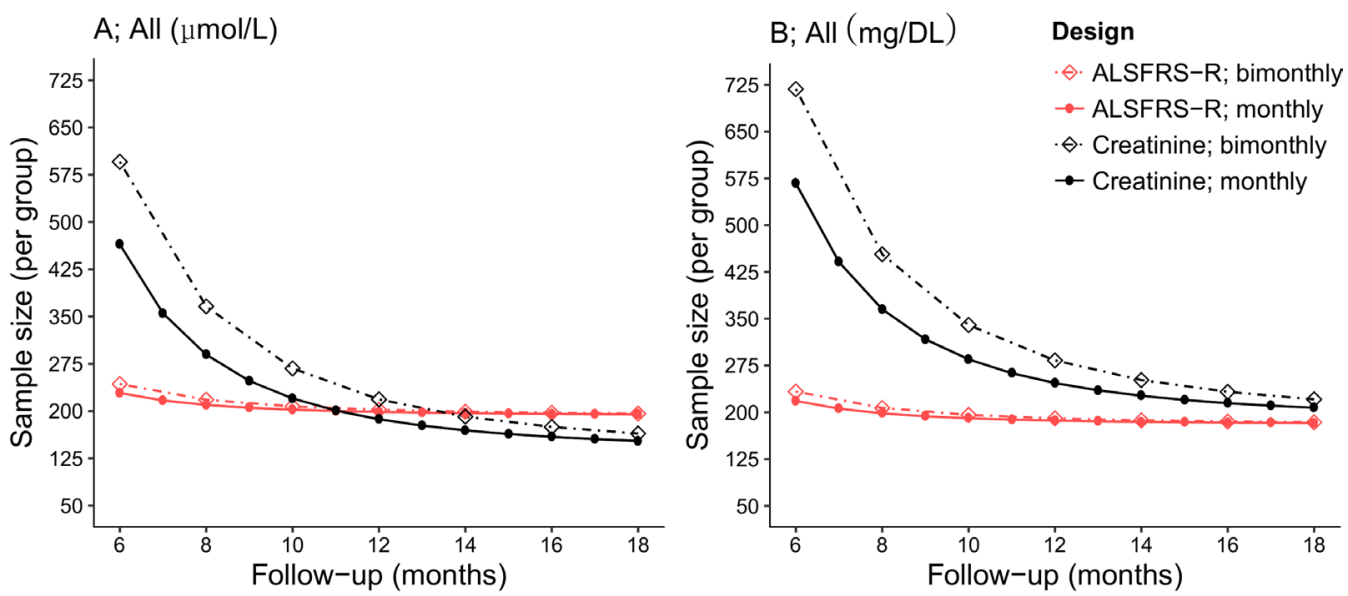

C; Spinal $(\mu \mathrm{mol} / \mathrm{L})$
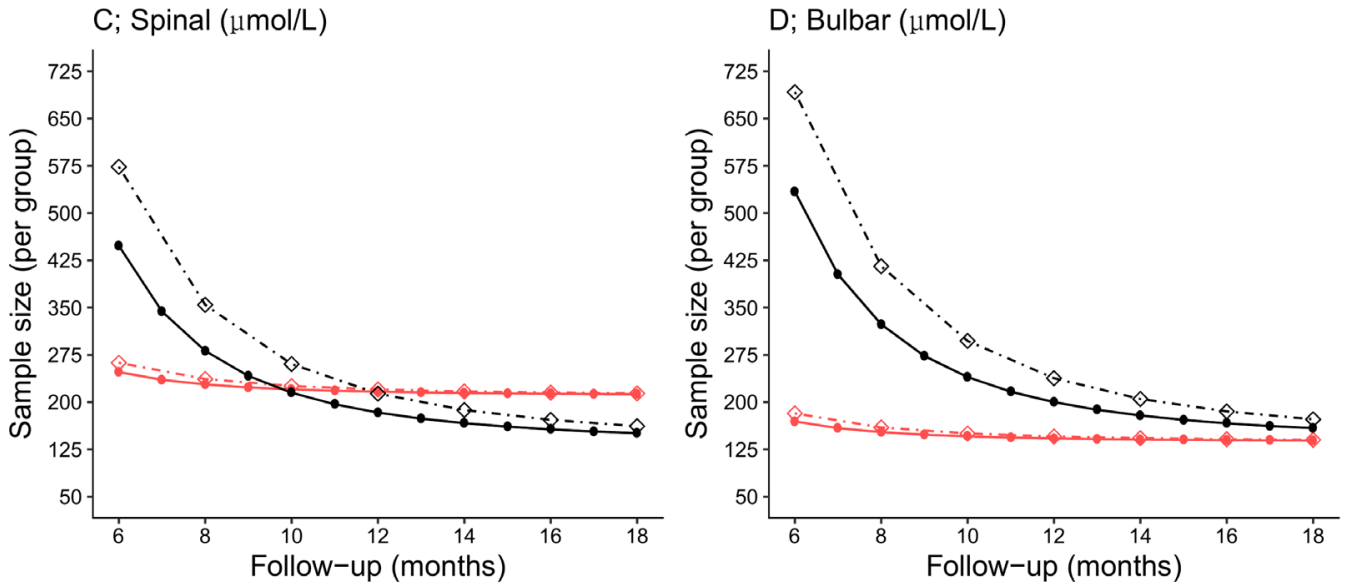

Figure 3 Sample size and power calculations for plasma creatinine and the ALSFRS-R. Sample size calculations were performed for the ALSFRS-R and plasma creatinine, with varying total follow-up durations (ranging 6-18 months) and with either bimonthly or monthly return visits. All calculations were based on an expected standardised 30\% reduction in slope during follow-up, with a two-sided alpha of 5\% and power of $90 \%$. For short trials (up to 10 months), the smaller between-patient variability at baseline and relatively faster rate of decline of the ALSFRS-R (see online supplementary eTable 1) resulted in smaller sample sizes. For longer trials (>10 months), sample size calculations for plasma creatinine, when determined in micromoles per litre, resulted in smaller sample sizes due to the lower variability between patients in rate of decline (A). When plasma creatinine levels were determined in micrograms per decilitre, this advantage was lost (B). The utility of plasma creatinine seems higher in spinal-onset patients (C) as compared with bulbar-onset patients (D). ALSFRS-R, amyotrophic lateral sclerosis functional rating scale-revised.

strong prognostic factor, it could be used for patient selection, subgroup analysis, as risk stratum or to provide additional insights into therapeutic effects, even for shorter phase I/II trials. The sensitivity of the ALSFRS-R may be improved by separating subdomains; however, this would pose significant difficulties for trial design (ie, specification of primary endpoint, sample size calculation and expectations of treatment effects), which are currently not well investigated.

As a final note, the ultimate aim of surrogate biomarkers is to replace the clinical endpoint (mortality). However, a strong association between the marker and the clinical endpoint is, in itself, not enough. For a biomarker to be regarded as a true surrogate outcome, it is vital that any treatment effect at the endpoint is directly reflected in the marker and vice versa. ${ }^{28}$ In this study, we showed the strong associations between functional decline, muscle strength and mortality. It is only by prospective use in future trials that one can determine whether plasma creatinine might act as true surrogate outcome and whether it accurately reflects the treatment effect. More importantly, prospective use may overcome possible regulatory hurdles generated by using plasma creatinine as efficacy endpoint.
In conclusion, in this paper we show the feasibility of monitoring ALS disease progression during clinical trials using plasma creatinine levels. Plasma creatinine is an inexpensive and easily accessible marker that exhibits considerably less variability between patients with ALS over time and correlates strongly with survival and functional outcomes. Plasma creatinine can, therefore, potentially increase the power to detect treatment effects and has the potency to serve as surrogate outcome measure in future ALS clinical trials.

Contributors RPAvE: study concept, design, analysis, interpretation of data and drafting manuscript. MJCE: study concept, design, analysis and interpretation of data. TAF: acquisition of data, critical revision of manuscript for intellectual content. SN: analysis and critical revision of manuscript for intellectual content. JHV: study concept, critical revision of manuscript for intellectual content. LHvdB: study supervision and critical revision of manuscript for intellectual content.

Disclaimer RPAvE reports no disclosures, MJCE reports no disclosures, TAF is an employee of Biogen Idec and hold shares in Biogen Idec, SN reports no disclosures, JV reports no disclosures, LHvd serves on scientific advisory boards for the Prinses Beatrix Spierfonds, Thierry Latran Foundation, Biogen Idec and Cytokinetics; received an educational grant from Baxter International; serves on the editorial board of Amyotrophic Lateral Sclerosis and the Journal of Neurology, Neurosurgery and Psychiatry; and receives research support from the Prinses Beatrix Spierfonds, Netherlands ALS Foundation, The European Community's Health Seventh Framework 
Programme (grant agreement $n^{\circ}$ 259867), The Netherlands Organization for Health Research and Development (Vici Scheme, JPND (SOPHIA,STRENGTH)).

Competing interests None declared.

Patient consent Obtained.

Ethics approval Obtained.

Provenance and peer review Not commissioned; externally peer reviewed.

(c) Article author(s) (or their employer(s) unless otherwise stated in the text of the article) 2018. All rights reserved. No commercial use is permitted unless otherwise expressly granted.

\section{REFERENCES}

1 Bacci ED, Staniewska D, Coyne KS, et al. Item response theory analysis of the amyotrophic lateral sclerosis ffunctional rating scale-revised in the pooled resource open-access ALS clinical trials database. Amyotroph Lateral Scler Frontotemporal Degener 2016;17:157-67.

2 Rutkove SB. Clinical measures of disease progression in amyotrophic lateral sclerosis. Neurotherapeutics 2015;12:384-93.

3 Franchignoni F, Mora G, Giordano A, et al. Evidence of multidimensionality in the ALSFRS-R Scale: a critical appraisal on its measurement properties using rasch analysis. J Neurol Neurosurg Psychiatry 2013;84:1340-5.

4 Weir CJ, Walley RJ. Statistical evaluation of biomarkers as surrogate endpoints: a literature review. Stat Med 2006;25:183-203.

5 Biomarkers definitions working G. Biomarkers and surrogate endpoints: preferred definitions and conceptual framework. Clin Pharmacol Ther 2001;69:89-95.

6 Baxmann AC, Ahmed MS, Marques NC, et al. Influence of muscle mass and physical activity on serum and urinary creatinine and serum cystatin C. Clin J Am Soc Nephrol 2008:3:348-54.

7 Patel SS, Molnar MZ, Tayek JA, et al. Serum creatinine as a marker of muscle mass in chronic kidney disease: results of a cross-sectional study and review of literature. J Cachexia Sarcopenia Muscle 2013;4:19-29.

8 Schutte JE, Longhurst JC, Gaffney FA, et al. Total plasma creatinine: an accurate measure of total striated muscle mass. J Appl Physiol Respir Environ Exerc Physiol 1981;51:762-6.

9 Patin F, Corcia P, Madji Hounoum B, et al. Biological follow-up in amyotrophic lateral sclerosis: decrease in creatinine levels and increase in ferritin levels predict poor prognosis. Eur J Neurol 2015;22:1385-90.

10 Chen X, Guo X, Huang R, et al. An exploratory study of serum creatinine levels in patients with amyotrophic lateral sclerosis. Neurol Sci 2014;35:1591-7.

11 Chiò A, Calvo A, Bovio G, et al. Amyotrophic lateral sclerosis outcome measures and the role of albumin and creatinine: a population-based study. JAMA Neurol 2014;71:1134-42.
12 Ikeda K, Hirayama T, Takazawa T, et al. Relationships between disease progression and serum levels of lipid, urate, creatinine and ferritin in Japanese patients with amyotrophic lateral sclerosis: a cross-sectional study. Intern Med 2012;51:1501-8.

13 Lawton KA, Brown MV, Alexander D, et al. Plasma metabolomic biomarker panel to distinguish patients with amyotrophic lateral sclerosis from disease mimics. Amyotroph Lateral Scler Frontotemporal Degener 2014;15:362-70.

14 Lawton KA, Cudkowicz ME, Brown MV, et al. Biochemical alterations associated with ALS. Amyotroph Lateral Scler 2012;13:110-8.

15 Atassi N, Berry J, Shui A, et al. The PRO-ACT database: design, initial analyses, and predictive features. Neurology 2014;83:1719-25.

16 Lunetta C, Lizio A, Melazzini MG, et al. Amyotrophic Lateral Sclerosis Survival Score (ALS-SS): simple scoring system for early prediction of patient survival. Amyotroph Lateral Scler Frontotemporal Degener 2015;17:93-100.

17 Paillisse C, Lacomblez L, Dib M, et al. Prognostic factors for survival in amyotrophic lateral sclerosis patients treated with riluzole. Amyotroph Lateral Scler Other Motor Neuron Disord 2005;6:37-44.

18 Küffner R, Zach N, Norel R, et al. Crowdsourced analysis of clinical trial data to predict amyotrophic lateral sclerosis progression. Nat Biotechnol 2015;33:51-7.

19 Bozik ME, Mitsumoto H, Brooks BR, et al. A post hoc analysis of subgroup outcomes and creatinine in the phase III clinical trial (EMPOWER) of dexpramipexole in ALS. Amyotroph Lateral Scler Frontotemporal Degener 2014;15:406-13.

20 Verstraete E, Veldink JH, Huisman MH, et al. Lithium lacks effect on survival in amyotrophic lateral sclerosis: a phase Illb randomised sequential trial. J Neurol Neurosurg Psychiatry 2012;83:557-64.

21 Cudkowicz ME, van den Berg LH, Shefner JM, et al. Dexpramipexole versus placebo for patients with amyotrophic lateral sclerosis (EMPOWER): a randomised, doubleblind, phase 3 trial. Lancet Neurol 2013;12:1059-67.

22 Rizopoulos D. Joint models for longitudinal and time-to-event data: with applications in R. Boca Raton: CRC Press, 2012.

23 Bates D, Mächler M, Bolker B, et al. Fitting Linear Mixed-Effects Models Using Ime4. J Stat Softw 2015;67:1-48.

24 Diggle P. Analysis of longitudinal data. Oxford: Oxford University Press, 2013.

25 Kollewe K, Mauss U, Krampfl K, et al. ALSFRS-R score and its ratio: a useful predictor for ALS-progression. J Neurol Sci 2008;275:69-73.

26 Shefner JM, Liu D, Leitner ML, et al. Quantitative strength testing in ALS clinical trials. Neurology 2016;87:617-624.

27 Miller TM, Pestronk A, David W, et al. An antisense oligonucleotide against SOD1 delivered intrathecally for patients with SOD1 familial amyotrophic lateral sclerosis: a phase 1, randomised, first-in-man study. Lancet Neurol 2013;12:435-42.

28 Gosho M, Nagashima K, Sato Y. Study designs and statistical analyses for biomarker research. Sensors 2012:12:8966-86. 